\title{
Retrieval of a tracheobronchial blood clot with a Yankauer suction catheter in complete airway obstruction
}

\author{
Sharad Kaushik', Gaurav Jain', Namrata Gupta', Lokesh Kumar Saini', Girish Sindhwani ${ }^{2}$ \\ Departments of ${ }^{1}$ Anaesthesiology and ${ }^{2}$ Pulmonary Medicine, All India Institute of Medical Sciences, Rishikesh, India
}

\section{Dear Editor:}

Tracheobronchial clot-induced airway obstruction is a life-threatening situation, requiring emergent intervention and expertise to prevent a catastrophic outcome. We report successful resuscitation of a kidney disease patient who developed complete airway obstruction and consequent cardiac arrest due to a large tracheobronchial blood clot during emergency bronchoscopy for continued hemoptysis.

We received a call for intubation of a 30-year-old male undergoing emergency bronchoscopy (day 5) for hemoptysis under general anesthesia. The patient had a history of kidney disease (details unavailable), hypertension for 4 years (Amlodipine $5 \mathrm{mg}$ twice a day), and recurrent symptoms suggesting urinary tract infection (UTI) but was not on any medical follow-up protocol. The patient was admitted (day 1 ) for fever with chills and rigor for 4 days and decreased urination and bilateral foot swelling for 15 days. After baseline investigations, the patient was diagnosed with chronic kidney disease, hypertension, and UTI and started on broad-spectrum antibiotics, a fluid/salt restricted diet, packed red blood cell (2 units) transfusions, and hemodialysis. On chest skiagram, mediastinal lymphadenopathy was suspected. A subsequent computed tomographic scan of the thorax and abdomen confirmed it as necrotizing mediastinal-retroperitoneal lymphadenopathy with centrilobular nodules in the right middle lobe (Figure 1). The patient received two cycles of hemodialysis. On day 4 , after a negative coronavirus disease 2019 (COVID-19) report, endobronchial ultrasound was performed through a flexible bronchoscopy (FB), and fine-needle aspirate (three times through a $21-G$ needle) samples were obtained from the right paratracheal lymph nodes ( $4 \mathrm{R}$ position) under local anesthesia. The patient had mild post-procedure bleeding but was otherwise stable. Later, the patient experienced an episode of mild hemoptysis. Therefore, tranexamic acid $1 \mathrm{~g}$ intravenously (IV) stat was administered, followed by $500 \mathrm{mg}$ IV three times a day, fresh frozen plasma (one unit), and desmopressin nasal spray 2 puffs twice a day, and the right lateral resting position was advised. However, hemoptysis increased in severity, so the patient was rushed for emergency bronchoscopy under local anesthesia on day 5 . However, the procedure was halted due to patient hypoxemia, and the anesthesia team sent a call for intubation.

Upon arrival, the vitals were heart rate 128/min, blood pressure 114/68 mm Hg, oxygen saturation $\left(\mathrm{SpO}_{2}\right) 85 \%$ on $6 \mathrm{~L}$ of oxygen through a facemask, sinus rhythm, and normother-

\section{Letter to the Editor}

Received: August 19, 2020

Revised: October 7, 2020

Accepted: December 9, 2020

Corresponding author

Gaurav Jain

Department of Anaesthesiology,

All India Institute of Medical

Sciences, Virbhadra Marg, Rishikesh,

Uttarakhand 249203, India

Tel: +91-8808631209

Fax: +91-135-2460994

E-mail: gauravhld@gmail.com

Copyright (@) 2021 The Korean Society of Critical Care Medicine

This is an Open Access article distributed under the terms of Creative Attributions Non-Commercial License (https:// creativecommons.org/li-censes/by-nc/4.0/ which permits unrestricted noncommercial use, distribution, and reproduction in any medium, provided the original work is properly cited. 

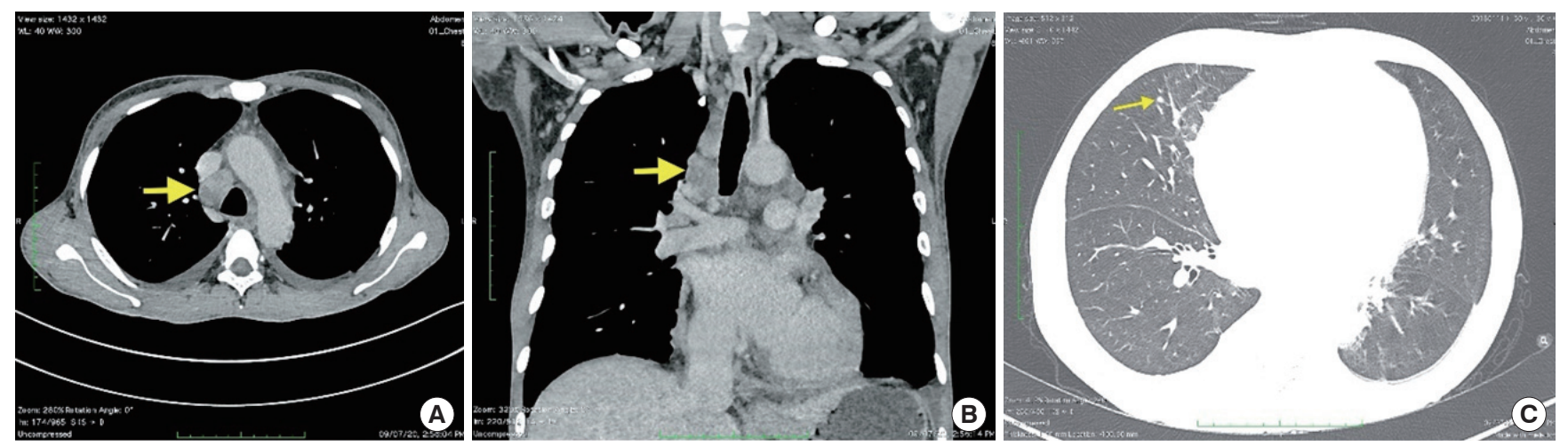

Figure 1. Computed tomography scan of the thorax. Mediastinal window showing necrotic lymphadenopathy of paratracheal lymph nodes (arrows) under axial (A) and coronal section (B). Lung window showing centrilobular nodules (arrow) in the right middle lobe under axial section (C).

mia. The patient was intubated by rapid sequence intubation using fentanyl $2 \mu \mathrm{g} / \mathrm{kg}$ IV, ketamine $1.5 \mathrm{mg} / \mathrm{kg} \mathrm{IV}$, and rocuronium $1 \mathrm{mg} / \mathrm{kg}$ IV. The trachea was intubated with an 8.0 size endotracheal tube (ETT), and manual ventilation with isoflurane $(0.5 \%-2 \%)$ and $100 \%$ fraction of inspired oxygen was initiated. Over the next $10-15$ minutes, the $\mathrm{SpO}_{2}$ improved to $100 \%$. Next, FB was introduced into the ETT. Upon first glance, both fresh and clotted blood were completely occluding the right bronchus, occupying the carina, and obscuring the left bronchus. We attempted endoscopic clot removal by a cryoprobe, but after a few failed attempts and persistent blood oozing, bronchoscopy with a rigid bronchoscope (RB) was proposed (Supplementary Video 1). Therefore, we extubated the patient and introduced an RB into the trachea. Ventilation was provided through a port in the RB. After multiple failed attempts to retrieve the clots, continuous oozing of blood, and gradual increases in clot size, $\mathrm{SpO}_{2}$ began to decrease. Therefore, we immediately removed the $\mathrm{RB}$ and initiated mask ventilation. However, we experienced extreme resistance in the reservoir bag and could not achieve chest expansion. Meanwhile, the $\mathrm{SpO}_{2}$ continued to drop, and the patient developed pulseless sinus bradycardia. Therefore, cardiopulmonary resuscitation (CPR) was started, adrenaline $1 \mathrm{mg}$ IV stat was given, and attempted ventilation through a facemask was continued. After 2 minutes of CPR, return of spontaneous circulation was achieved. On laryngoscopy, we observed a blood clot occluding the airway below the glottis. First, we attempted to remove it through a flexible suction catheter (14 Fr), but failed. Therefore, we introduced a Yankauer rigid suction catheter into the larynx, but again failed to remove the clot. However, the clot then was retrieved en bloc by slowly dragging it up under continuous negative suction pressure of the Yankauer catheter (Figure 2). Afterward, we inserted a 7.0-sized ETT into the trachea and observed adequate chest rise on manual

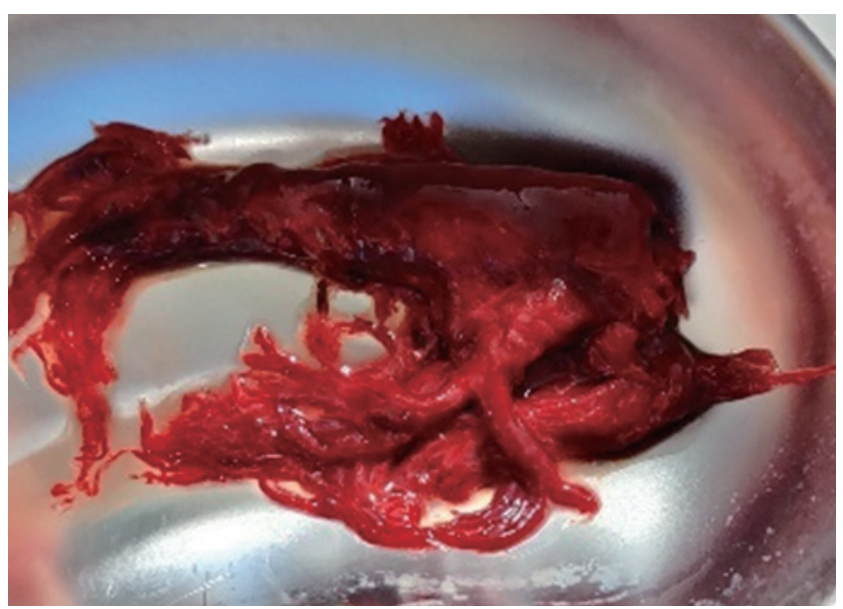

Figure 2. Massive tracheobronchial clot with visible primary and secondary divisions of the bronchial tree.

ventilation without increased airway resistance. We inserted the FB into the ETT and observed a clear tracheobronchial tree without residual bleeding (Supplementary Video 1). The vitals returned to normal within 5 minutes. The patient received adrenaline nebulization and hydrocortisone $100 \mathrm{mg}$ IV stat, was shifted to the intensive care unit under ventilation support, and was extubated the next day without any complications.

Tracheobronchial bleeding and clot formation are known complications of interventional airway procedures. Multiple attempts to remove the clot may further aggravate the airway edema, cause fresh bleeding, and increase the size of the blood clot due to ongoing trauma to the mucosa by the suction catheter or probe [1]. Ongoing bleeding further limits the visibility of the FB in such situations. The RB offers a larger bore size for better visibility and suction but compromises patient ventilation. The obstructing clot may act in a ball-valve mechanism to cause selective expiratory obstruction, which 
may precipitate as tension pneumothorax or may lead to complete airway obstruction with subsequent hemodynamic collapse and even mortality [1]. The alternative options include tracheal suction, cryoprobe, forceps, fibrinolytic agents, or embolectomy catheter [1-4]. In this patient, such a sequence precipitated as a life-threatening situation. The tracheobronchial blood clot was so large that it could not be retrieved either through cryoprobe, forceps, or suction under FB or RB guidance or sucked out with a flexible or Yankauer catheter. We attempted to pull the clot slowly through the Yankauer catheter. The rigid structure of the Yankauer catheter and its better suction pressure allowed the blood clot to attach to the catheter end, and we ultimately retrieved the entire clot in a single piece with the catheter. In conclusion, the Yankauer catheter might be an effective lifesaving tool for removing tracheobronchial blood clots in a crisis where conventional modalities are ineffective.

\section{CONFLICT OF INTEREST}

No potential conflict of interest relevant to this article was reported.

\section{ORCID}

Sharad Kaushik

Gaurav Jain

https://orcid.org/0000-0001-9437-0923

Namrata Gupta https://orcid.org/0000-0002-1205-7237 https://orcid.org/0000-0002-7720-3784

Lokesh Kumar Saini https://orcid.org/0000-0002-6972-3966
Girish Sindhwani

https://orcid.org/0000-0003-2765-2343

\section{AUTHOR CONTRIBUTIONS}

Data curation: SK, GJ, LKS, GS. Investigation: all authors. Resources, Supervision, Validation: GJ. Writing-original draft: SK, GJ. Writing-review \& editing: SK, GJ, LKS, GS.

\section{SUPPLEMENTARY MATERIALS}

Supplementary materials can be found via https://doi.org/ 10.4266/acc.2020.00675.

\section{REFERENCES}

1. Bodenham AR. Removal of obstructing blood clot from the lower airway: an alternative suction technique. Anaesthesia 2002;57:40-3.

2. Wong B, Souza C. The use of a Fogarty catheter in a case of an endobronchial blood clot in a laryngectomy patient. Int J Head Neck Surg 2015;6:118-20.

3. Inoue H, Ito J, Uchida H, Morita M, Masuda T, Yamaya K, et al. Lower airway obstruction due to a massive clot resulting from late bleeding following mini-tracheostomy tube insertion and subsequent clot removal and re-intubation. JA Clin Rep 2017;3:16.

4. Sehgal IS, Dhooria S, Behera D, Agarwal R. Use of cryoprobe for removal of a large tracheobronchial foreign body during flexible bronchoscopy. Lung India 2016;33:543-5. 
Supplementary Video 1. Flexible bronchoscopy view showing the attempts to retrieve blood clots by cryoprobe, and the clear tracheobronchial tree view after clot removal by the Yankauer suction catheter. 